\title{
Técnica mínimamente invasiva en paciente con multimorbilidad crónica y hernia inguinal complicada: reporte de caso
}

\author{
A minimally invasive technique in a patient with chronic multimorbidity and complicated \\ inguinal hernia: case report
}

René M. Palacios Huatuco', Diana A. Pantoja Pachajoa', Julián E. Liaño', Facundo I. Mandojana ', Alejandro M. Doniquian ${ }^{1}$

\section{Resumen}

El paciente con multimorbilidad crónica forma parte de una población que se ha incrementado en los últimos años. La hernia inguinal incarcerada representa una emergencia. El abordaje transabdominal preperitoneal (TAPP) presenta ventajas para evaluar el contenido de la hernia. Se presenta el caso de un hombre de 77 años con múltiples comorbilidades y cirugías abdominales previas, que se presentó con cuadro de oclusión intestinal. En tomografía computada: asa de intestino delgado incarcerada en región inguinal. Se observó asas intestinales dilatadas con cambio de calibre en región inguinal izquierda. Se retiró asa intestinal atascada en hernia directa. Se disecó defecto herniario y se colocó malla de 10 x $15 \mathrm{~cm}$ en espacio preperitoneal. La técnica TAPP es eficaz y segura para la reparación de hernias complicadas en pacientes con multimorbilidad crónica, en manos de cirujanos experimentados.

Palabras clave: multimorbilidad; enfermedad crónica; hernia incarcerada; laparoscopia; TAPP.

\begin{abstract}
The patient with chronic multimorbidity is part of a population that has increased in recent years. Incarcerated inguinal hernia represents an emergency. The preperitoneal transabdominal approach (TAPP) has advantages to evaluate the content of the hernia. A 77-years-old man with multiple comorbidities and previous abdominal surgeries presented with intestinal occlusion. Computed tomography: small bowel loop incarcerated in the inguinal region. The cavity is inspected by observing dilated intestinal loops with a change of caliber in the left inguinal region. The intestinal loop is removed observing a direct hernia. The hernia defect is repaired, and $10 \times 15 \mathrm{~cm}$ mesh is placed in the preperitoneal space. The TAPP technique is effective and safe for the repair of complicated hernias in patients with chronic multimorbidity, in the hands of experienced surgeons.
\end{abstract}

Keywords: multimorbidity; chronic disease; incarcerated hernia; laparoscopy; TAPP.

Fecha de envío: 2020-07-08- Fecha de aceptación: 2020-11-24

\section{Introducción}

En países desarrollados el ingreso hospitalario de pacientes con multimorbilidad crónica se ha convertido en un fenómeno creciente en los últimos años (Silber et al., 2018). A medida que la población envejece la multimorbilidad se incrementa, entendiéndose la misma como la presencia de 2 o más enfermedades crónicas, físicas o mentales en un mismo individuo, ocurridas al mismo tiempo, donde una no es complicación de la otra (Struckmann et al., 2018; Barros Urzua J et al., 2019). En Argentina, los pacientes con multimorbilidad generan una importante demanda en diferentes niveles asistenciales, siendo estas enfermedades crónicas responsables del 73,4\% de las muertes, de acuerdo con los últimos datos registrados por la $4^{\text {ta }}$ Encuesta Nacional de Factores de Riesgo 2018 (INDEC, 2018).

Una de las patologías quirúrgicas con mayor incidencia en adultos mayores es la hernia inguinal, pudiendo generar efectos nocivos si no es tratada a tiempo, con una incidencia que aumenta de 11 por cada 10000 habitantes en personas entre 16 y 24 años, a 200 por cada 10000 habitantes en personas mayores de 75 años (Sartelli et al., 2013). El riesgo de presentar una hernia en la población general es del 27 - 43\% en hombres y 3 - 6\% en mujeres (Köckerling \& Simons, 2018).

(1) Servicio de Cirugía General, Clínica Universitaria Reina Fabiola, Universidad Católica de Córdoba, Córdoba Capital, Argentina.

Autor de correspondencia: manuelpalacioshuatuco@gmail.com 
Palacios et al.

\section{Caso clínico}

Hombre de 77 años, con antecedentes patológicos de obesidad grado I (IMC: 33 kg/m2), hipertensión arterial, dislipidemia, insuficiencia renal crónica, arritmia no filiada y antecedentes quirúrgicos de hernioplastía inguinal bilateral convencional, colecistectomía y hepatectomía izquierda convencional. Consultó por servicio de guardia central, presentando dolor abdominal de 24 horas de evolución, generalizado, tipo cólico, de intensidad moderada, con distensión, constipación y obstipación del mismo tiempo de evolución, asociado a náuseas y vómitos. En el ingreso, los signos vitales fueron: frecuencia cardíaca 100/min, frecuencia respiratoria 22/min, presión arterial 160/100 mmHg, y al examen físico presentó el abdomen asimétrico (incisión Mercedes Benz), distendido, ruidos hidroaéreos escasos, a tensión, timpánico, dolor generalizado, sin signos de peritonismo. Región inguinal sin particularidades. En los exámenes de laboratorio se encontró hemoglobina de 14,7 g/dL, hematocrito 43,1\%, leucocitos 15 $\mathrm{mil} / \mathrm{mm}^{3}$ (valor normal: $3,8-10 \mathrm{mil} / \mathrm{mm}^{3}$ ), proteína c reactiva $37 \mathrm{mg} / \mathrm{l}$ (valor normal: <5), creatinina $3 \mathrm{mg} / \mathrm{dL}$ (valor normal: 0,74 -1,22), ácido láctico 1,8 mmol/l (valor normal 0,5 - 2,22), KPTT $37 \mathrm{seg}$, tiempo de protrombina 80\%, recuento de plaquetas $150 \mathrm{mil} / \mathrm{ul}$, gases arteriales con $\mathrm{pH} 7,4, \mathrm{pCO}_{2} 37, \mathrm{HCO}_{3}$ 23. Se solicitó radiografía de abdomen que informó niveles hidroaéreos periféricos. Se agregó tomografía computada de abdomen y pelvis: asa de intestino delgado que protruye a través de la pared abdominal en región inguinal izquierda con nivel hidroaéreo. Se realizó la valoración preoperatoria del paciente frágil: la puntuación del índice de comorbilidad de Charlson fue de 5 y fue clasificado como ASA III. Se aplicó el riesgo quirúrgico del Colegio Americano de Cirujanos (NSQIP - ACS), los resultados se muestran en la tabla 1. Se indicó exploración laparoscópica por cuadro de oclusión intestinal, con sospecha de hernia inguinal complicada.

Se realizó abordaje transabdominal preperitoneal (TAPP) y se inspeccionó la cavidad abdominal, que presentó poco espacio por la presencia de asas intestinales dilatadas, con cambio de calibre en región inguinal izquierda. Se retiró asa intestinal observando hernia directa. Se procedió a realizar tratamiento del defecto con apertura del peritoneo. Se confeccionó flap superior e inferior. Se disecó espacio preperitoneal ampliamente, con reducción de saco herniario directo delimitando anillo de 5 por $4 \mathrm{~cm}$. Se colocó malla de polipropileno multifilamento de alto peso de 10 por 15 $\mathrm{cm}$ fijándola con 5 a 6 agrafes absorbibles. Se procedió a cerrar peritoneo. No se presentaron intercurrencias y el tiempo operatorio estimado fue de 90 minutos. El paciente requirió internación en la unidad de cuidados coronarios en el postoperatorio inmediato por bloqueo auriculoventricular de $1^{\text {er }}$ grado, con bradicardia sinusal, que se controló sin medicación extraordinaria.

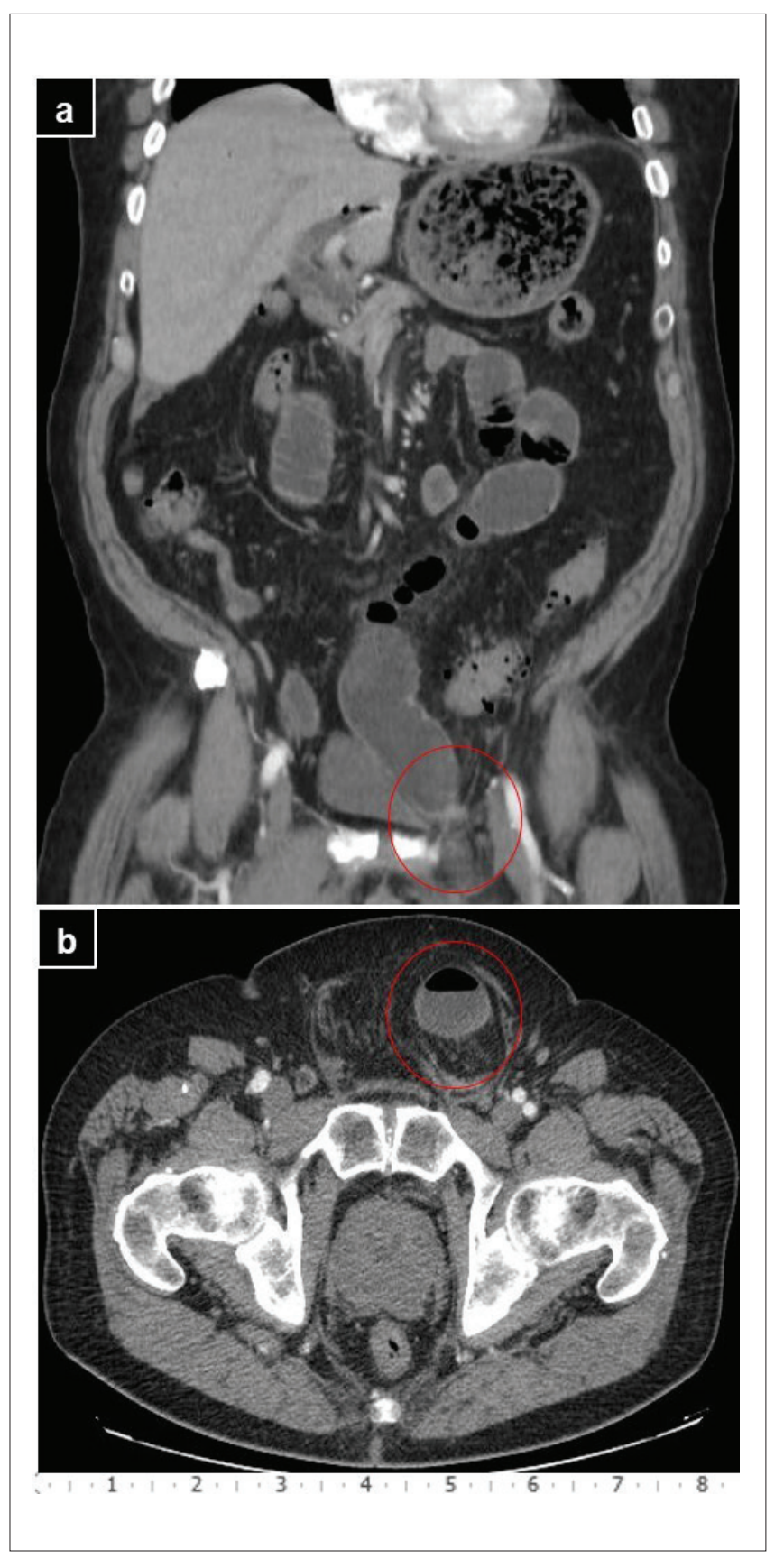

Figura 1: Tomografía computada de abdomen y pelvis. a) Corte coronal: se observa dilatación retrógrada de asas intestinales, protruyendo hacia región inguinal izquierda (círculo rojo). b) Corte axial: intestino delgado incarcerado con nivel hidroaéreo (círculo rojo). 
Palacios et al.

Tabla 1: Características de riesgo quirúrgico de NSQIP de ACS. Estima la posibilidad de un resultado desfavorable (como una complicación o muerte) después de la cirugía.

\begin{tabular}{l} 
Resultados \\
Complicaciones severas \\
1. Neumonía \\
2. Complicación cardíaca \\
3. Infección del sitio operatorio \\
4. Tromboembolismo venoso \\
5. Retorno al quirófano \\
6. Sepsis \\
\hline
\end{tabular}
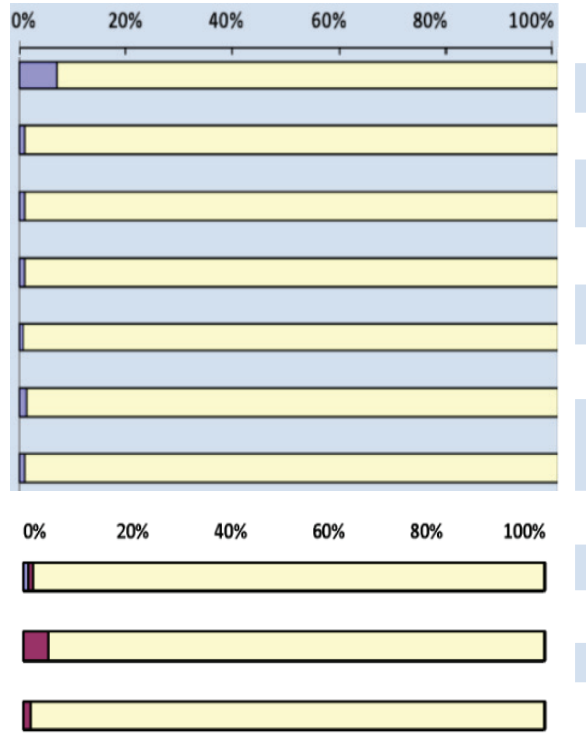

Estadía hospitalaria estimada: $\mathbf{2}$ días
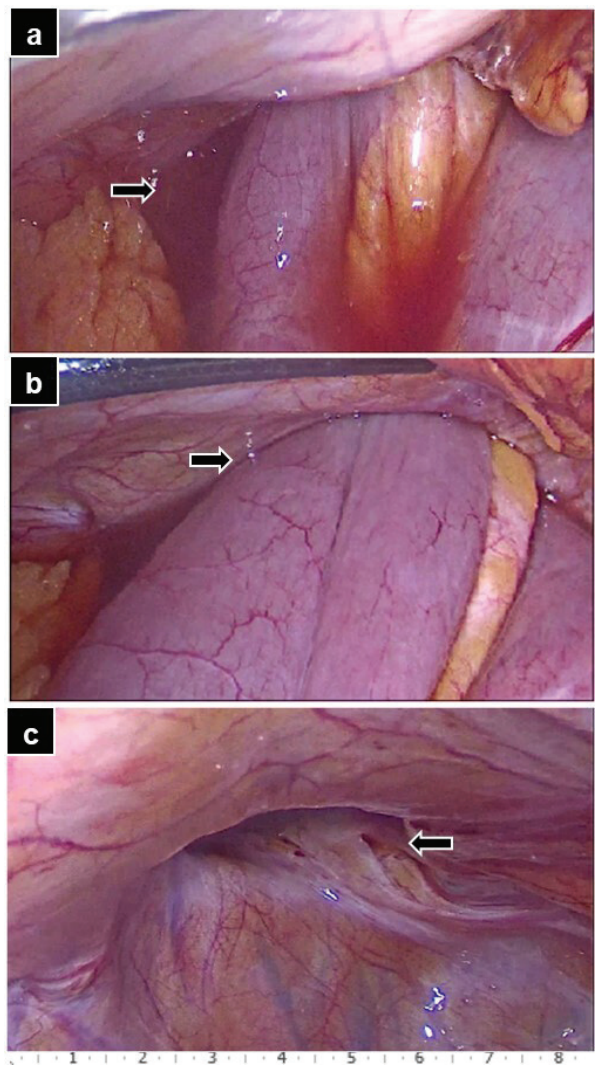

Figura 2: a) En la inspección abdominal la cavidad presentó un espacio reducido debido a la distensión intestinal. Se identificó líquido inflamatorio y asa de intestino delgado incarcerada por su borde antimesentérico (flecha negra). b) Reducción con maniobras suaves del contenido intestinal con la utilización de pinzas atraumáticas laparoscópicas (flecha negra). c) Hallazgo de defecto en pared abdominal anterior, compatible con hern ia inguinal izquierda directa de $5 \times 4 \mathrm{~cm}$ (flecha negra).

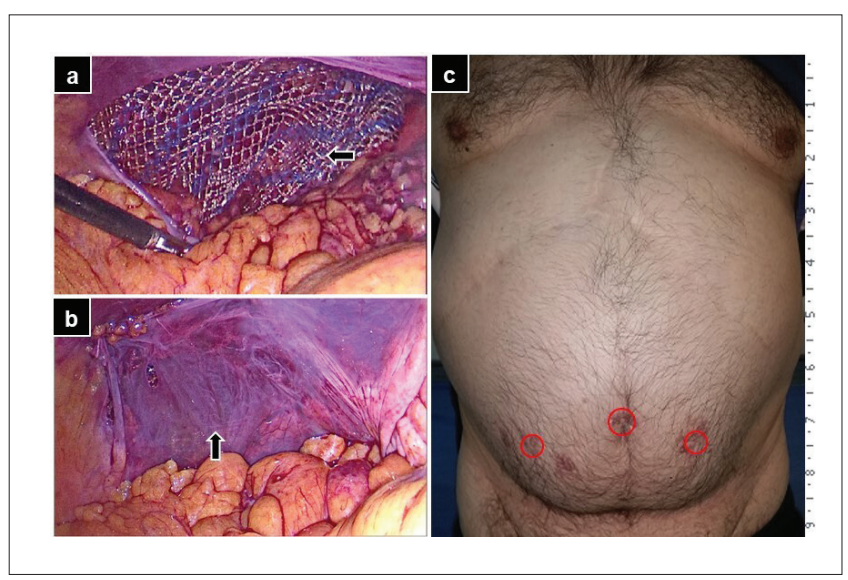

Figura 3: a) Malla de polipropileno multifilamento posicionada en espacio preperitoneal, cubriendo el defecto parietal (flecha negra). b) Visión intraabdominal de región inguinal izquierda reparada y cierre de peritoneo (flecha negra). c) Obesidad abdominal con cicatrices quirúrgicas por puertos laparoscópicos (círculos rojos).

Posteriormente presentó evolución favorable en sala común, con restitución del tránsito intestinal, tolero dieta el $2^{\text {do }}$ día postoperatorio y fue dado de alta al $3^{\text {er }}$ día de la cirugía por control de múltiples comorbilidades. Se controló a los 30 días, 3, 6, 12 y 18 meses del alta. No registró otras complicaciones durante el seguimiento postoperatorio.

\section{Discusión}

En áreas quirúrgicas la incidencia de pacientes con multimorbilidad crónica se ha incrementado con el tiempo asociándose directamente a la edad, formando parte de una población con especial fragilidad clínica e importante deterioro funcional, generando una mayor necesidad de recursos hospitalarios (Hewitt et al., 2016). 
Los adultos mayores presentan alto riesgo de morbi-mortalidad cuando se someten a cirugías electivas o de emergencia, debido al procedimiento quirúrgico en sí o a las condiciones del paciente, por lo que merecen una atención individualizada (Dewan et al., 2012).

La hernia inguinal complicada (incarcerada y/o estrangulada) tiene efectos perjudiciales severos, como obstrucción intestinal, translocación bacteriana y necrosis de la pared intestinal (lo que puede provocar una perforación intestinal), requiriendo una intervención quirúrgica con carácter de emergencia (Leibl et al., 2001). En pacientes jóvenes se presenta en el 5 - 6\%, mientras que en adultos mayores en el 12 - 20\% (Sartelli et al., 2013). Esta situación representa un problema debido a que estos pacientes requieren un mayor número de días de hospitalización y en el $20 \%$, necesitarán de nutrición parenteral domiciliaria (Legnani et al., 2008). Sin embargo, la edad no puede predecir estos eventos en forma aislada, por lo que deben evaluarse múltiples factores, incluido el estado funcional antes de la cirugía y la presencia de comorbilidades (Ackland et al., 2010).

Se han publicado guías de práctica clínica como la del Colegio Americano de Cirujanos (NSQIP - ACS, American College of Surgeons National Surgical Quality Improvement Program) (Bilimoria et al., 2013), la Sociedad Americana de Anestesiología (ASA) y distintas escalas de valoración de morbilidades para pacientes sometidos a procedimientos quirúrgicos (Lee et al., 1999; Ackland et al., 2010; Fleisher et al., 2014).

El principal predictor de complicaciones postoperatorias es la severidad de las comorbilidades y los cambios asociados al envejecimiento (inmovilización, privación sensorial, reducción de la ingesta calórica y cambios en la reserva fisiológica), permitiendo que los adultos mayores sometidos a cirugía sean más vulnerables al estrés producido por el procedimiento (Charlson et al., 2008). En este caso realizamos la valoración preoperatoria aplicando distintos sistemas de puntuación y encontramos un paciente con enfermedad sistémica severa (ASA III), una supervivencia estimada a los 10 años del 21,36\% (Charlson), y un riesgo de complicaciones severas del 7\% (NSQIP - ACS), lo que nos informó que estábamos ante un paciente que requería una mayor vigilancia.

El objetivo de la valoración preoperatoria es identificar los factores de riesgo asociados a las complicaciones quirúrgicas y recomendar el acompañamiento y tratamiento individualizado a lo largo de todas las fases del cuidado perioperatorio para minimizar dichos riesgos (Charlson et al., 2008; Fleisher et al., 2014). Consideramos que esto podría desempeñar un papel fundamental en la toma de decisiones en el tratamiento para pacientes de edad avanzada y en mal estado general.
En los últimos años, el enfoque laparoscópico en el abordaje de la hernia inguinal complicada ha sido descrito por varios autores y controvertido entre los cirujanos (Rebuffat et al., 2006). Se demostró que la laparoscopía es un procedimiento seguro y preciso, teniendo ventajas como: menor dolor postoperatorio, menor tasa de complicaciones (infecciones, hematomas), ofreciendo una reinserción más temprana a las actividades habituales, ventajas que aportan un beneficio particularmente en adultos mayores con comorbilidades de alto riesgo (Legnani et al., 2008).

Como terapéutica se decidió optar por el abordaje TAPP, técnica laparoscópica que presenta ventajas anteriormente mencionadas en comparación con la cirugía abierta, así como también, es utilizada en el tratamiento simultáneo de la hernia inguinal bilateral (Sartelli et al., 2013). En la literatura se considera que este abordaje es particularmente útil para evaluar y tratar el contenido de la hernia, siendo factible de realizarse en un contexto de emergencia en adultos mayores (Leibl et al., 2001). La reparación de la hernia inguinal sin contaminación del campo operatorio, se puede realizar por laparoscopia después del reposicionamiento de los órganos incarcerados, ofreciendo la oportunidad de corrección de la hernia y la visión y control de la cavidad abdominal, permitiendo un tratamiento definitivo del contenido de la hernia con posibilidad de resecar el intestino (si fuera necesario) o reparar una hernia contralateral oculta, presente en el 11,2 - 50\% de los casos (Rebuffat et al., 2006). En contraposición, en la cirugía abierta, el contenido de la hernia incarcerada podría regresar a la cavidad abdominal, debido al uso de narcóticos y relajantes musculares durante la inducción anestésica, por lo que el contenido reintroducido siempre genera dudas entre los cirujanos de la necesidad de realizar la exploración abdominal convencional (Legnani et al., 2008; Bittner et al., 2015).

Las complicaciones postoperatorias se emplean como un indicador de calidad para evaluar un procedimiento quirúrgico, para seleccionar las alternativas terapéuticas frente a un caso particular y para comparar los resultados entre centros o en un mismo centro en períodos diferentes (Clavien et al., 2009). En la actualidad, los sistemas de clasificación de complicaciones postoperatorias más utilizados son la clasificación de Clavien - Dindo (CD), que divide a las complicaciones en grados de acuerdo a la forma de resolución de las mismas y el índice comprehensivo de complicaciones (ICC), que está basado en la clasificación anterior, y toma en cuenta para su cálculo todos los eventos postoperatorios con su respectiva gravedad (Clavien et al., 2009; Slankamenac et al., 2013). En este paciente se registró complicaciones menores de acuerdo con la clasificación CD (Grado I) y un valor de 8,7 según el ICC, lo que refleja objetivamente los eventos negativos después de una cirugía. 
Si bien la técnica TAPP ofrece beneficios por sobre las técnicas abiertas, representa un procedimiento más desafiante en el paciente adulto mayor con multimorbilidad crónica, en comparación con el abordaje convencional, requiriendo un entrenamiento laparoscópico adecuado (Bittner, 2016; Mancini et al., 2019; Bittner \& Schwarz, 2019).

\section{Conclusión}

El abordaje TAPP en pacientes con multimorbilidad crónica es posible con un riesgo relativamente bajo de morbi-mortalidad en cirugías limpias/contaminadas como es el caso de la resección intestinal, siendo una técnica eficaz y segura en manos de cirujanos experimentados.

\section{Responsabilidades éticas}

Confidencialidad de los datos. Los autores declaran que han seguido los protocolos de su centro de trabajo sobre la publicación de datos de pacientes.

Conflictos de interés. Ninguno.

Fuentes de Apoyo. Ninguna.

\section{Referencias}

Ackland GL, Harris S, Ziabari Y, Grocott M \& Mythen M. (2010). Revised cardiac risk index and postoperative morbidity after elective orthopaedic surgery: a prospective cohort study. British journal of anaesthesia 105, 744- 752.

Association Task Force on practice guidelines. Journal of the American College of Cardiology 64, 77-137.

Barros Urzua J, Zamorano Pichard P, Varela Yuraszeck, T, Irazoqui Soto E \& Téllez A. (2019). Cambios en el modelo de atención de personas con multimorbilidad crónica: una revisión narrativa. ARS MEDICA Revista De Ciencias Médicas 44, 35-40.

Bilimoria KY, Liu Y, Paruch JL, Zhou L, Kmiecik TE, Ko CY, et al. (2013). Development and evaluation of the universal ACS NSQIP surgical risk calculator: a decision aid and informed consent tool for patients and surgeons. Journal of the American College of Surgeons 217, 833- 842.

Bittner R, Montgomery MA, Arregui E, Bansal V, Bingener J, Bisgaard T, et al. (2015). Update of guidelines on laparoscopic (TAPP) and endoscopic (TEP) treatment of inguinal hernia (International Endohernia Society). Surgical endoscopy 29, 289-321.

Bittner JG 4th. (2016). Incarcerated/Strangulated Hernia: Open or Laparoscopic?. Advances in surgery 50, 67-78.
Bittner R. \& Schwarz J. (2019). Primary Unilateral Not Complicated Inguinal Hernia: Our Choice of TAPP, Why, Results and Review of Literature. Hernia: the journal of hernias and abdominal wall surgery 23, 417-428.

Charlson ME, Charlson RE, Paterson JC, Marinopoulos SS, Briggs WM. \& Hollenberg JP. (2008). The Charlson comorbidity index is adapted to predict costs of chronic disease in primary care patients. Journal of clinical epidemiology 61, 1234-1240.

Clavien PA, Barkun J, de Oliveira ML, Vauthey JN, Dindo D, Schulick RD, et al. (2009). The Clavien-Dindo classification of surgical complications: five-year experience. Annals of surgery 250, 187- 196.

Deeba S, Purkayastha P, Paraskevas T, Athanadious A, Darzi A \& Zacharakis E. (2009). Laparoscopic approach to incarcerated and strangulated inguinal hernias. Journal of the Society of Laparoendoscopic Surgeons 13, 327-331.

Dewan SK, Zheng SB \& Xia SJ. (2012). Preoperative geriatric assessment: comprehensive, multidisciplinary and proactive. European journal of internal medicine 23, 487- 494.

Fleisher LA, Fleischmann KE, Auerbach AD, Barnason SA, Beckman JA, Bozkurt B, et al. (2014). American College of Cardiology; American Heart Association. 2014 ACC/AHA guideline on perioperative cardiovascular evaluation and management of patients undergoing noncardiac surgery: a report of the American College of Cardiology/American Heart

Hewitt J, McCormack C, Tay HS, Greig M, Law J, Tay A, et al. (2016). Prevalence of multimorbidity and its association with outcomes in older emergency general surgical patients: an observational study. BMJ Open 6, e010126.

INDEC Encuesta Nacional de Factores de Riesgo. Resultados definitivos. Instituto Nacional de Estadística y Censos (2018) - Secretaría de Gobierno de Salud de la Nación. Argentina. Disponible en https:// www.argentina.gob.ar/noticias/salud-publico-el-informe-completo-de-la-4deg-encuesta-nacional-de-factores-de-riesgo el 21 de octubre de 2019.

Köckerling F. \& Simons M. (2018). Current Concepts of Inguinal Hernia Repair. Visceral Medicine 34, 145-150.

Lee TH, Marcantonio ER, Mangione CM, Thomas EJ, Polanczyk CA, Cook EF, et al. (1999). Derivation and prospective validation of a simple index for prediction of cardiac risk of major noncardiac surgery. Circulation 100, 1043-1049. 


\section{Palacios et al.}

Legnani GL, Rasini M, Pastori S \& Sarli D. (2008). Laparoscopic Trans-Peritoneal Hernioplasty (TAPP) for the Acute Management of Strangulated Inguino-Crural Hernias: A Report of Nine Cases. Hernia : the journal of hernias and abdominal wall surgery 12, 185-8.

Leibl BJ, Schmedt CG, Kraft K, Kraft B \& Bittner R. (2001). Laparoscopic transperitoneal hernia repair of incarcerated hernias: is it feasible? Results of a prospective study. Surgical endoscopy 15, 1179-1183

Mancini R, Pattaro G \& Spaziani E. (2019). Laparoscopic Trans-Abdominal Pre-Peritoneal (TAPP) Surgery for Incarcerated Inguinal Hernia Repair. Hernia : the journal of hernias and abdominal wall surgery 23, 261-266.

Rebuffat C, Galli A, Scalambra MS \& Balsamo F. (2006). Laparoscopic Repair of Strangulated Hernias. Surgical endoscopy 20, 131-4.
Sartelli M, Coccolini F, Van Ramshorst GH, Campanelli G, Mandalà V, Ansaloni L, et al. (2013). WSES guidelines for emergency repair of complicated abdominal wall hernias. World journal of emergency surgery 1, 8 - 50 .

Silber J, Reiter J, Rosenbaum P, Zhao Q, Small D, Niknam B, et al. (2018). Defining Multimorbidity in Older Surgical Patients. Medical Care 56, 701-710.

Slankamenac K, Graf R, Barkun J, Puhan M \& Clavien P. (2013). The comprehensive complication index: a novel continuous scale to measure surgical morbidity. Annals of surgery 258, 1-7.

Struckmann V, Leitjen F, van Ginneken E, Kraus M, Reiss M, Spranger A, et al. (2018). Relevant models and elements of integrated care for multimorbidity:Results of a scoping review. Health Policy 122, 23-35. 